\title{
Implementação, acesso e uso das práticas integrativas e complementares no Sistema Único de Saúde: revisão da literatura
}

\author{
Implementation, access and use of integrative and complementary \\ practices in the unified health system: a literature review
}

Ludmila de Oliveira Ruela (http://orcid.org/0000-0001-9071-539X) ${ }^{1}$

Caroline de Castro Moura (http://orcid.org/0000-0003-1224-7177) ${ }^{2}$

Clícia Valim Côrtes Gradim (http://orcid.org/0000-0002-1852-2646) ${ }^{3}$

Juliana Stefanello (https://orcid.org/0000-0003-3926-0144) ${ }^{1}$

Denise Hollanda Iunes (http://orcid.org/0000-0003-1396-9980) ${ }^{3}$

Rogério Ramos do Prado (https://orcid.org/0000-0003-2767-3283) ${ }^{4}$

${ }^{1}$ Escola de Enfermagem de Ribeirão Preto,

Universidade de São Paulo. Av. dos Bandeirantes 3900,

Monte Alegre. 14040-902 Ribeirão Preto SP Brasil. ludmilaoliveira@usp.br ${ }^{2}$ Escola de Enfermagem, Universidade Federal de Minas Gerais. Belo Horizonte MG Brasil. 3 Escola de Enfermagem, Universidade Federal de Alfenas. Alfenas MG Brasil. ${ }^{4}$ Diretoria de Extensão e Assuntos Comunitários, Universidade José do Rosário Vellano. Alfenas MG Brasil.

\begin{abstract}
In Brazil, the Integrative and Complementary Practices (ICP) achieved greater visibility after the establishment of the National Integrative and Complementary Practices Policy (NICPP) in 2006. However, there are still gaps in the general setting of these practices. Thus, this study aimed to analyze the implementation, access and use of ICPs in the Brazilian Unified Health System (SUS) after the establishment of this policy. We performed an integrative literature review, guided by the question: "What is the current setting of implementation, access and use of ICPs within the SUS?", in the Virtual Health Library (BVS), the U.S. National Library of Medicine and in the Web of Science, with descriptors "Sistema Unico de Saúde" / "Unified Health System" AND "Terapias Complementares" / "Complementary Therapies". The analysis of papers gave rise to four categories for discussion: "The ICP approach in the SUS: main practices used"; "Access to ICPs: Primary Health Care as a gateway"; "Current implementation scenario of ICPs: the preparation of health services and professionals for to implement ICPs"; "Main advances in the use of ICPs and future challenges". We have observed that ICPs are bashfully offered and that data available are scarce, despite the positive impacts on users and services that have embraced their use.
\end{abstract}

Key words Unified Health System, Complementary therapies
Resumo No Brasil, as Práticas Integrativas e Complementares (PIC) tiveram maior visibilidade após a criação da Política Nacional de Práticas Integrativas e Complementares, em 2006. Contudo, ainda existem lacunas sobre o cenário geral dessas práticas. O objetivo deste estudo foi analisar a implementação, o acesso e o uso das PIC no Sistema Único de Saúde (SUS) após a implantação da política. Foi realizada uma revisão integrativa da literatura, guiada pela questão: "Qual o atual cenário de implementação, acesso e utilização das PIC no âmbito do SUS?", na Biblioteca Virtual em Saúde, na US National Library of Medicine e na Web of Science, com os descritores "Sistema Único de Saúde"/"Unified Health System" AND "Terapias complementares"/"Complementary Therapies". Da análise dos artigos, emergiram quatro categorias de discussão: "A abordagem das PIC no SUS: principais práticas usadas"; "O acesso às PIC: a Atenção Básica à Saúde como porta de entrada"; "Atual cenário de implementação das PIC: o preparo dos serviços e dos profissionais da saúde para a realização das PIC"; "Principais avanços no uso das PIC e desafios futuros". Observa-se que as PIC são oferecidas de forma tímida e os dados disponiveis são escassos, apesar dos reflexos positivos para os usuários e para os serviços que aderiram à sua utilização.

Palavras-chave Sistema Único de Saúde, Terapias complementares 


\section{Introdução}

Desde a década de 1990, o uso das Práticas Integrativas e Complementares (PIC) tem aumentado em proporções mundiais ${ }^{1}$. $\mathrm{O}$ seu crescimento e visibilidade ocorreram, principalmente, com estímulo da Organização Mundial de Saúde (OMS), em 2002, por meio da elaboração de um documento normativo para seus países membros. Este documento visa o desenvolvimento e a regulamentação de tais práticas nos serviços de saúde, bem como a ampliação do acesso, do uso racional e da avaliação da eficácia e da segurança de tais técnicas a partir de estudos científicos ${ }^{2}$.

Neste cenário, em 2006, o Ministério da Saúde (MS), por meio da Portaria no 971/2006, publicou a Política Nacional de Práticas Integrativas e Complementares (PNPIC) no Sistema Único de Saúde (SUS), com o intuito de garantir a integralidade nos serviços de saúde ${ }^{3}$. A partir de então, a oferta e o estímulo ao uso das PIC, como a fitoterapia, a homeopatia, a acupuntura, dentre outras, foi legitimada no SUS, ampliando a utilização dessas práticas ${ }^{4}$.

É importante ressaltar, que a implantação da PNPIC teve caráter político, técnico, econômico, social e cultural, uma vez que estabeleceu diretrizes nacionais para o uso das PIC, a partir de experiências e práticas já adotadas nos serviços de saúde que obtiveram resultados satisfatórios ${ }^{3}$. Tal fato possibilitou ainda mais a difusão dessas práticas em diversos pontos do país.

Nesse contexto, o Brasil tem se destacado como um dos 69 Estados-Membros da OMS que possuem políticas e estratégias específicas para o uso das PIC . Após a criação da PNPIC, 30\% dos municípios brasileiros adotaram regulamentação própria para o uso dessas terapias, o que indica um importante incremento das práticas na atenção à saúde; e a Atenção Básica à Saúde (ABS) é um dos principais ambientes para a sua aplicação ${ }^{4}$.

Diante disso, torna-se imperativo analisar o atual cenário de oferta dessas práticas no país, bem como o acesso a elas e sua utilização nos serviços de saúde pública. Assim, o objetivo desse estudo foi analisar a implementação, o acesso e o uso das PIC no SUS, por meio de revisão da literatura nacional publicada após a implantação da PNPIC.

\section{Método}

Trata-se de uma revisão integrativa da literatura, fundamentada nas etapas propostas por Whittemore e Knafl', com a seguinte questão norteadora: "Qual o atual cenário de implementação, acesso e utilização das Práticas Integrativas e Complementares no âmbito do Sistema Único de Saúde?".

Foram realizadas buscas na Literatura Latino-americana em Ciências da Saúde (LILACS), Base de Dados de Enfermagem (BDENF), HomeoIndex e Índice Bibliográfico Espanhol de Ciências de Saúde (IBECS) via Biblioteca Virtual em Saúde (BVS); na Medical Literature Analysis and Retrieval System Online (MEDLINE) via US National Library of Medicine (PUBMED) e na Web of Science, por dois pesquisadores independentes, com descritores padronizados extraídos dos Descritores em Ciências da Saúde (DECS) e o operador booliano AND, que resultaram na combinação: "Sistema Único de Saúde" / "Unified Health System” AND “Terapias complementares" / "Complementary Therapies".

Adotaram-se como critérios de inclusão artigos publicados no período de 2006 a 2017, nos idiomas português, inglês e espanhol, com o resumo disponível na base de dados, e que foram realizados em cenário nacional. Os estudos que não responderam à questão norteadora foram excluídos.

Primeiramente, os artigos foram selecionados, por dois pesquisadores independentes, pela leitura do título e do resumo, de acordo com a questão norteadora e com os critérios de elegibilidade. Após a seleção, eles foram lidos na íntegra e, para coleta e avaliação dos dados, aplicou-se um instrumento elaborado pelos pesquisadores, adaptado de Ursi ${ }^{7}$. Tal instrumento foi composto pelos itens: título do artigo; autores e ano de publicação; objetivo(s) do estudo; características metodológicas; nível de atenção em saúde (primário, secundário e terciário) em que o estudo foi realizado; resultados; e conclusões.

A Figura 1 apresenta o fluxograma de seleção dos artigos.

\section{Resultados}

O Quadro 1 apresenta as principais informações extraídas dos artigos selecionados.

Em relação ao tipo de PIC utilizadas, 23,52\% dos estudos abordaram a fitoterapia ${ }^{8-11}, 17,64 \%$ a homeopatia $^{12-14}, 5,90 \%$ a acupuntura ${ }^{15}$ e 52,94\% 

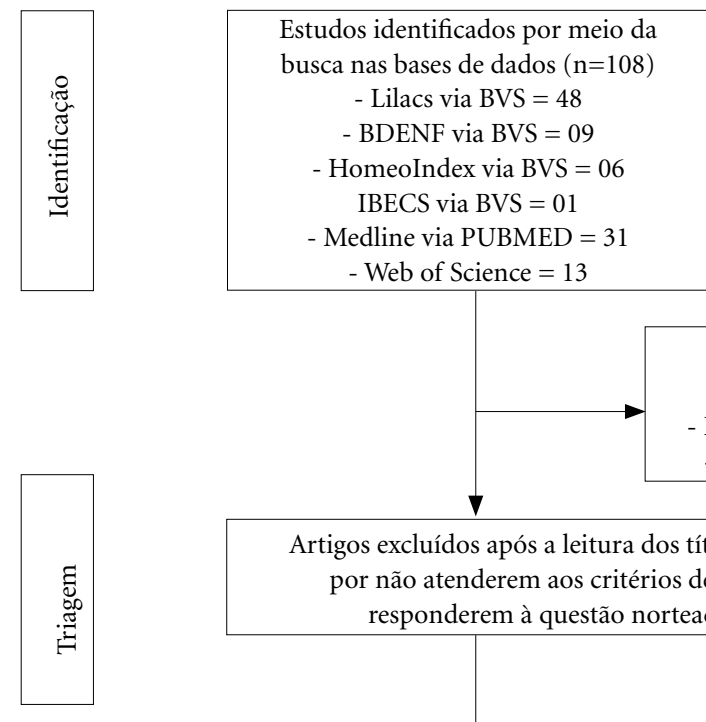

- Web of Science $=13$
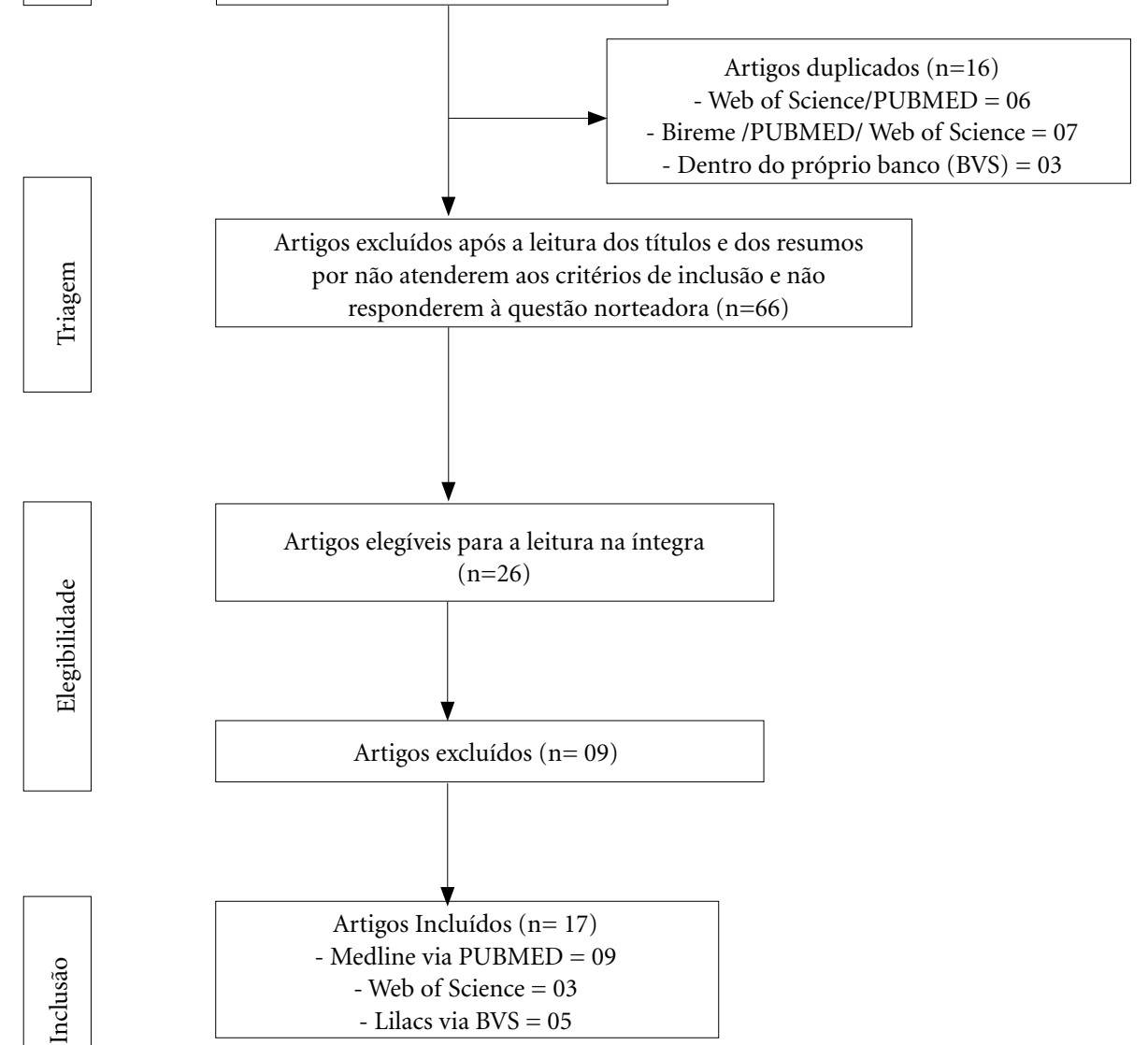

Figura 1. Fluxograma de seleção dos artigos.

Fonte: Dos autores.

dos estudos avaliaram as PIC de um modo ge$\mathrm{ral}^{16-24}$.

Referente ao nível de atenção onde as práticas foram realizadas, $52,94 \%$ ocorreu no nível primário de atenção $0^{8,10-12,16,19-21,24}$ e $17,65 \%$ no nível primário e/ou secundário ${ }^{15,22,23}$. Outros autores $(29,41 \%)$ fizeram a abordagem das práticas no cenário geral do SUS ${ }^{9,13,14,17,18}$. Identificou-se, ainda, que todos os artigos analisados apresentavam o nível IV de evidência. Além disso, os temas mais abordados foram: as principais PIC adotadas no SUS, o acesso a essas práticas, e o preparo dos serviços e dos profissionais da saúde para a implementação e utilização das mesmas. A partir desses temas, foram elaboradas as categorias de discussão. 


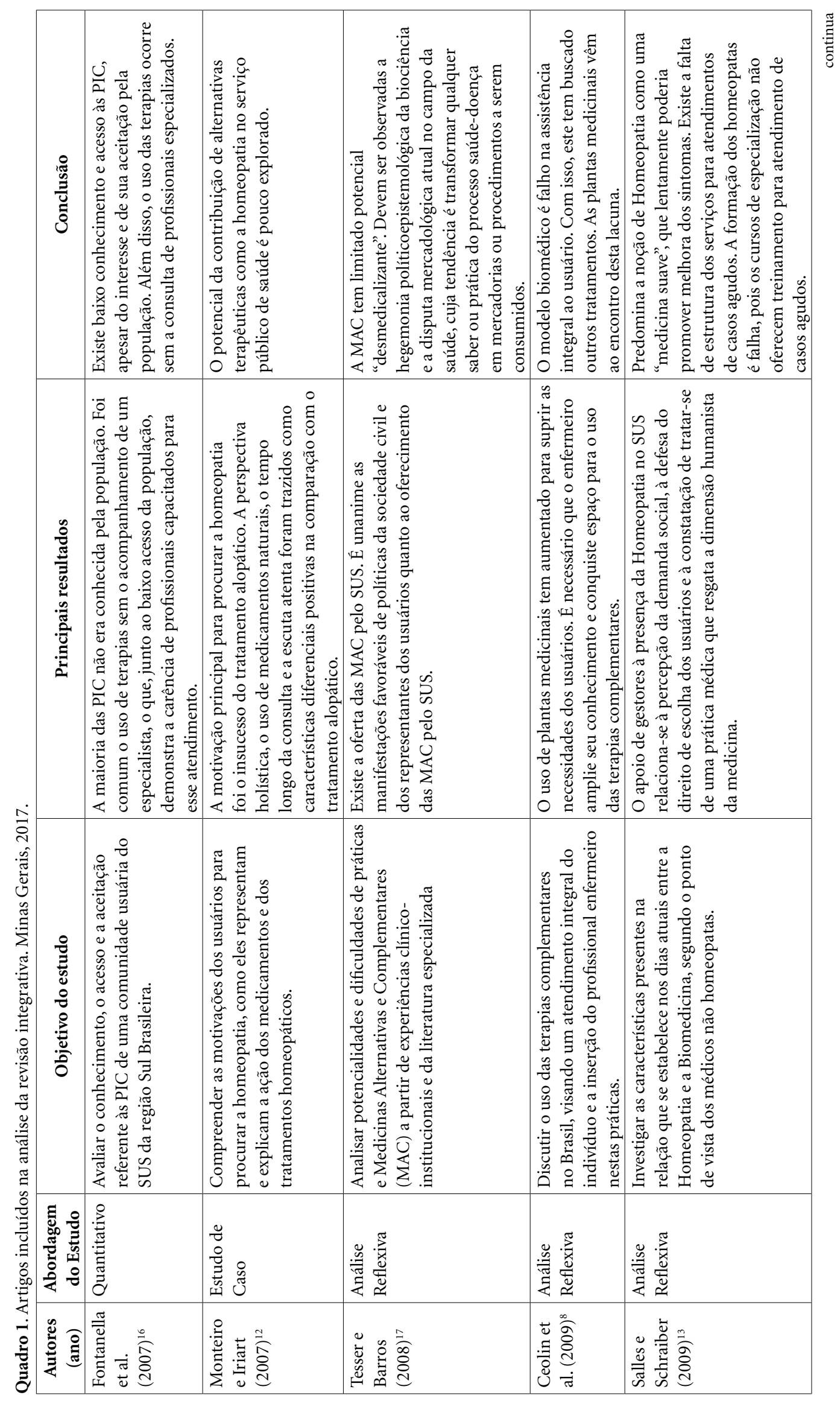




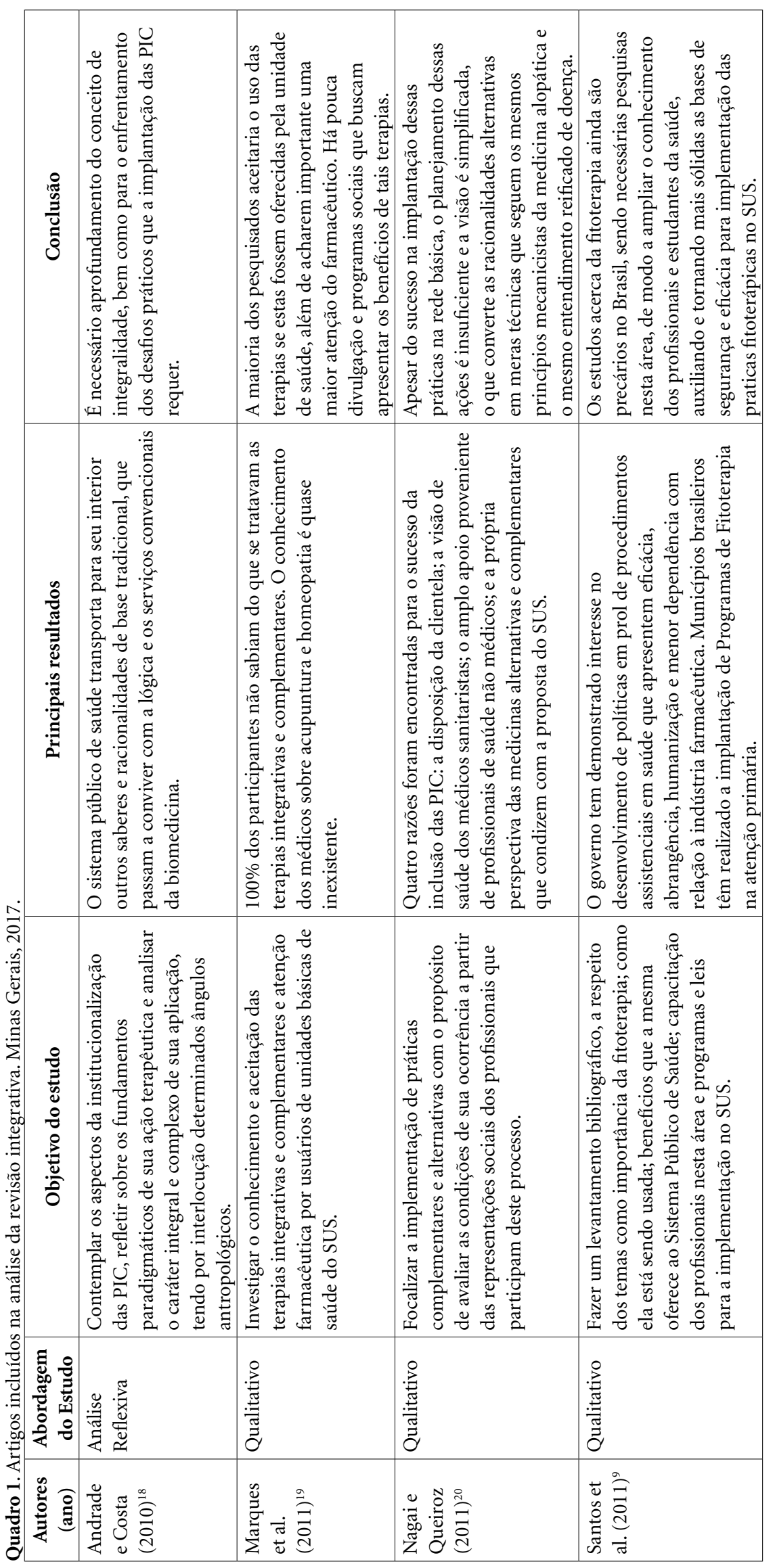




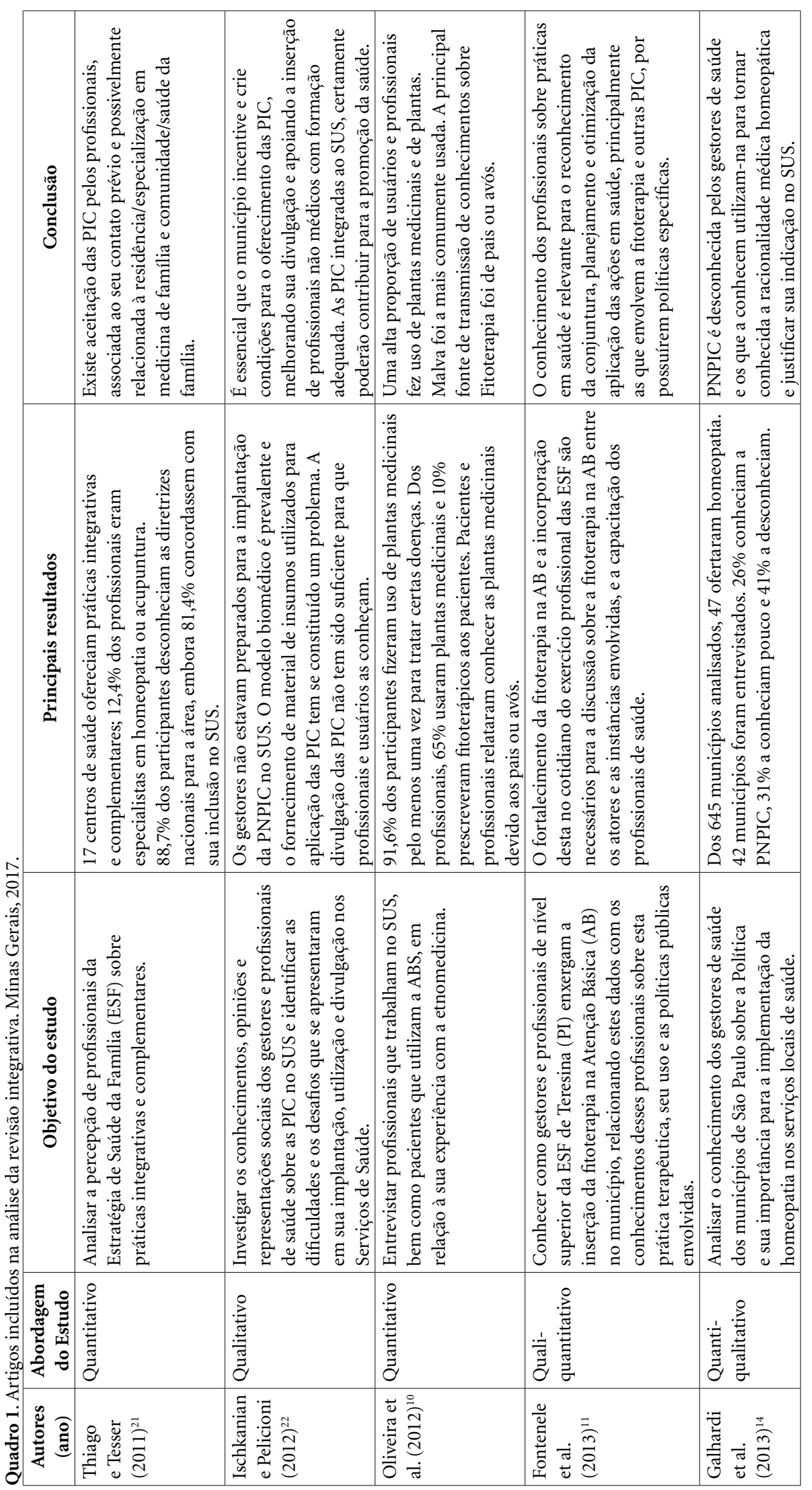




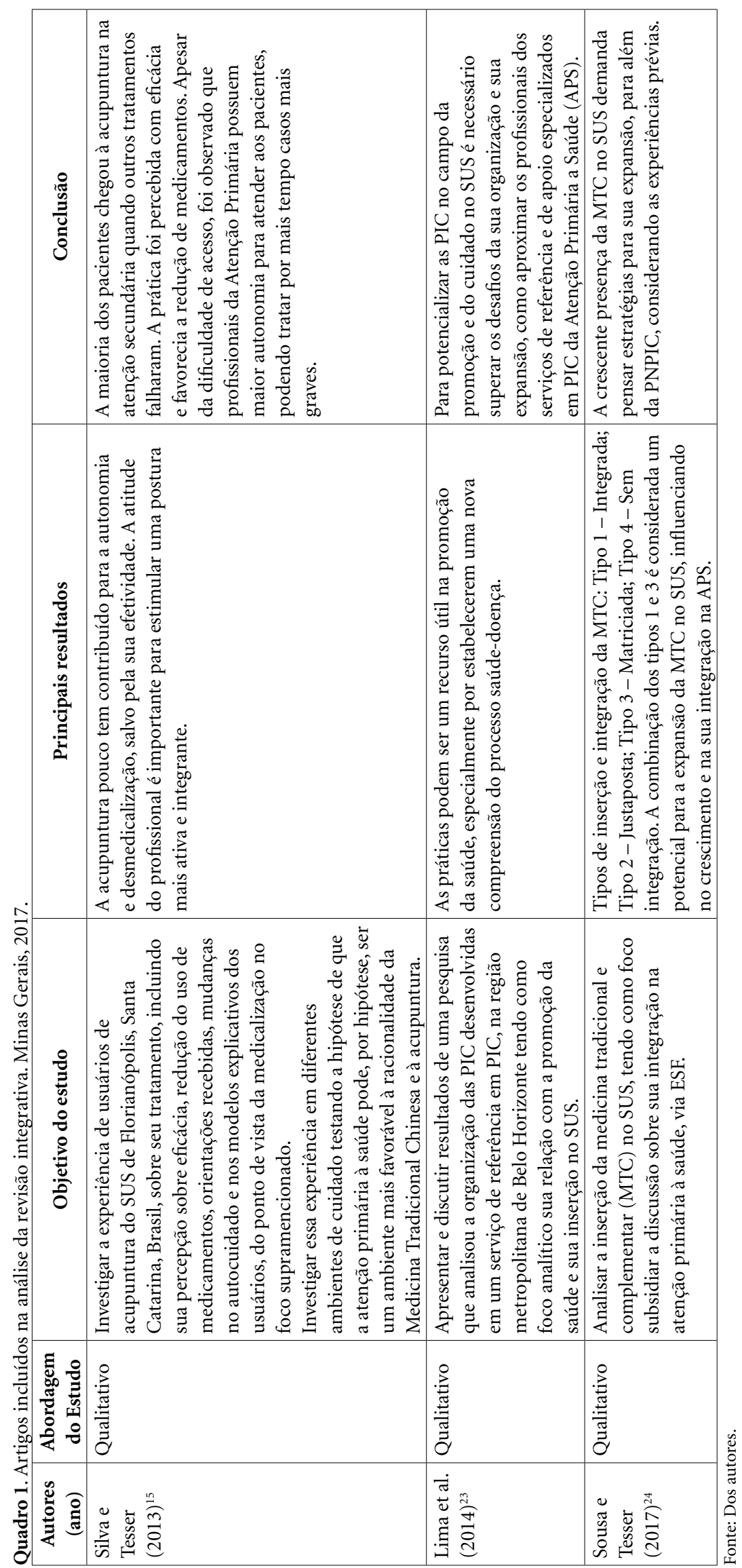




\section{Discussão}

\section{A abordagem das PIC no SUS: principais práticas usadas}

Inicialmente, a PNPIC elencava apenas cinco PIC em suas diretrizes para serem empregadas no SUS com o intuito de promover a recuperação, a manutenção e a prevenção da saúde dos usuários, além da cura de algumas doenças, são elas: a Medicina Tradicional Chinesa/Acupuntura; a Homeopatia; as Plantas Medicinais/Fitoterapia; o Termalismo/Crenoterapia; e a Medicina Antroposófica ${ }^{25}$. Entretanto, ao reconhecer a crescente utilização de outras práticas baseadas em conhecimentos tradicionais pela população de uma forma em geral, o MS incluiu, entre os anos de 2017 e 2018, novos recursos terapêuticos à PNPIC, por meio da Portaria $n^{\circ} 849 / 2017^{26} \mathrm{e} \mathrm{da}$ Portaria $n^{\circ} 702 / 2018^{27}$. Com as medidas, o SUS passou a ofertar, atualmente, 29 dessas práticas.

Diante das opções de PIC incentivadas pela política, os resultados deste estudo mostram que muitas dessas não foram abordadas pelos autores ou foram apenas citadas, sem aprofundamentos. Houve, com isso, o predomínio de estudos que abordaram várias práticas na mesma investigação, como por exemplo: a acupuntura, a homeopatia, a fitoterapia, entre outras, analisando a implantação e organização dessas e o conhecimento dos usuários e dos profissionais sobre as $\mathrm{PIC}^{16,19,20,22-}$ 24; bem como daqueles estudos que apontaram um contexto geral das terapias no SUS, sem fazer especificação das práticas usadas ${ }^{17,18,21}$. Assim, foi possível observar a escassez de estudos que aprofundassem o uso de algumas práticas, como o Termalismo/Crenoterapia e a medicina Antroposófica, apontando uma lacuna para a utilização dessas no SUS. Entretanto, isso pode ser um reflexo da baixa oferta de tais terapias nos serviços, o que impossibilita a discussão dessas práticas nos estudos analisados.

Dentre os estudos que analisaram práticas de maneira específica, destaca-se a utilização da fitoterapia $^{8-11}$ e da homeopatia ${ }^{12-14}$. A acupuntura foi investigada de modo isolado apenas por Silva e Tesser ${ }^{15}$. Entretanto, tanto a homeopatia quanto a acupuntura, mesmo nos estudos que analisaram várias práticas em conjunto, se destacaram como aquelas que apresentam maior adesão pelos usuários e maior oferta pelos serviços ${ }^{15,21,22}$. Esse fato vai ao encontro de dados apresentados pelo MS, em 2008, que demonstram maior interesse por parte do governo e da população nessas terapias quando comparadas às demais ${ }^{28}$.
Embora os mecanismos de ação da acupuntura $^{29,30} \mathrm{e}$ da homeopatia ${ }^{31,32}$ ainda não sejam totalmente claros e, por vezes, inconclusivos, os seus benefícios têm sido demonstrados em diferentes estudos, para diferentes enfermidades ${ }^{33-36}$. Com isso, a adesão a esses tratamentos é cada vez mais progressiva, de forma que $80 \%$ dos 129 países membros da OMS já reconhecem a acupuntura como um tratamento de saúde ${ }^{5}$, e a homeopatia é uma das PIC mais indicadas em países europeus, a exemplo da França ${ }^{37}$.

As PIC, em geral, podem ser vistas como uma importante estratégia de assistência à saúde, especialmente por considerarem a pessoa em sua integralidade, diferenciando-se do modelo biomédico $^{23}$. A procura pelas PIC dá-se, na maioria das vezes, por motivos complexos, que envolvem desde fatores como o baixo perfil de efeitos adversos, passando pelo efeito natural de estímulos à cura de dentro para fora; pela busca de complementação do tratamento alopático; pelo acolhimento e escuta qualificada realizada durante a consulta; assim como, pela compatibilidade de tais práticas com os valores, as crenças e a filosofia de saúde e de vida do usuário ${ }^{17,38}$. Além disso, elas podem ser percebidas como um potencial para redução no consumo de medicamentos ${ }^{15}$.

Tesser ${ }^{39}$ ressalta ainda que os motivos que levam os usuários a procurar tais tratamentos podem estar associados a fatores socioeconômicos importantes. Em países pobres a cultura local, o fácil acesso às práticas alternativas, $o$ alto custo da medicina convencional e a pouca oferta de recursos da biomedicina, facilitam a procura pela medicina complementar. Entretanto, em países ricos, a insatisfação com o modelo biomédico e os próprios benefícios das PIC são os fatores que incentivam essa procura.

\section{O acesso às PIC: a Atenção Básica à Saúde (ABS) como porta de entrada}

Uma vez que a ABS deve ser o primeiro contato e a porta de entrada do usuário para a rede de atenção à saúde, de acordo com a Política Nacional de Atenção Básica (PNAB $)^{40}$ é possível inferir que esse nível de atenção constitui locus privilegiado para a implementação das PIC no sistema público de saúde brasileiro. De fato, dados do MS apontam que as PIC são ofertadas, em sua grande maioria, nos serviços de atenção básica ${ }^{41}$.

Um estudo recente ${ }^{42}$, realizado em Florianópolis, apontou que normalmente os profissionais da ABS incentivavam o uso das PIC ainda durante a consulta com o paciente e iniciam o tratamento 
logo que possível, muitas vezes durante a própria consulta. Nesse sentido, o tratamento com as PIC pode se configurar, em alguns casos, como a abordagem inicial, sendo o tratamento convencional a segunda opção, se necessário, ou complementar à abordagem das PIC. Além disso, a disponibilidade das PIC nos serviços de ABS pode promover um maior diálogo dos profissionais com os usuários sobre qual terapia usar, a convencional ou as PIC, e isso pode ter efeito positivo neste contato ${ }^{42}$.

No presente estudo, alguns autores apontam serviços de atenção secundária ${ }^{15,22,23}$ como forma de acesso às PIC. Contudo, para a concretização desses locais como um campo de cuidado e oferta de tratamentos complementares, é necessária a aproximação dos profissionais de ambos os níveis de atenção, primário e secundário, para que estes se consolidem como uma rede de cuidados integrais e de acesso universal às PIC, considerando os princípios e fundamentos de cada uma das práticas $^{23}$.

Embora o uso de PIC em ambientes de atenção secundária e terciária seja mais restrito, observa-se uma tendência, ainda que tímida, para a sua utilização também nesses níveis, visto que 1.708 municípios brasileiros oferecem as PIC, sendo $78 \%$ na $A B S, 18 \%$ na atenção secundária e $4 \%$ na atenção terciária ${ }^{43}$. Contudo, ao considerar a $\mathrm{ABS}$ como o nível de atenção com a maior capacidade de desenvolver ações de prevenção e de recuperação da saúde, o uso das PIC nesses serviços é o mais indicado. Soma-se a isso o fato de que tais práticas não necessitam de recursos tecnológicos sofisticados, oferecem menores riscos de efeitos colaterais quando comparados aos tratamentos convencionais, e necessitam de menos recursos financeiros, o que torna a assistência em saúde menos onerosa e com qualidade, além de proporcionar resultados satisfatórios ${ }^{2,44}$.

Todavia, a dificuldade de acesso às PIC nos diversos níveis de atenção, principalmente no secundário e terciário, pode estar relacionada à falta de conhecimento dos profissionais sobre o uso dessas práticas. Além disso, ressalta-se o fato de que muitos destes não entendem a importância ou não têm habilidade adequada para indicar ou aplicar tais práticas ${ }^{16,18}$.

Apesar dessa dificuldade, a sua oferta nos serviços é aceita e esperada, sobretudo pelos usuá$\operatorname{rios}^{17,19}$. Desse modo, é observado um movimento dos municípios brasileiros para implantar o uso das PIC nos últimos anos?. Porém, é essencial que a gestão local incentive o fortalecimento e o uso dessas práticas e proporcione condições para que as mesmas sejam oferecidas à população, por meio de sua divulgação e apoio, seguindo as recomendações da PNPIC ${ }^{11,22}$.

\section{Atual cenário de implementação das PIC: o preparo dos serviços e dos profissionais da saúde para a realização das PIC}

Para o uso das PIC no SUS, os recursos humanos são essenciais. Nesse contexto, a formação profissional é considerada como uma importante lacuna para o sucesso da implementação das práticas $^{13,16,22}$. O desconhecimento da PNPIC, bem como das terapias abordadas na política dificulta a adesão, tanto de profissionais quanto dos serviços, na oferta das práticas ${ }^{13,14}$.

No Brasil, além de médicos, outros profissionais da saúde, como enfermeiros, fisioterapeutas, farmacêuticos, entre outros, são habilitados para o uso de diversas práticas estimuladas pela política ${ }^{3}$. Porém, a baixa adesão a especializações na área das intervenções complementares e a deficiência no ensino sobre as finalidades do uso das PIC, durante a formação, impedem melhor aperfeiçoamento dos profissionais da saúde ${ }^{9}$, embora muitos demonstrem interesse na capacitação e concordância com o uso das práticas nos servi$\operatorname{ços}^{21}$.

Uma das principais dificuldades apontadas pelos gestores para a implementação dessas terapias é a resistência por parte de alguns profissionais de saúde, atribuída à escassez de evidências científica e falta de apoio logístico e estrutural da gestão local ${ }^{11}$. Considera-se, portanto, este um importante problema, visto que a atitude positiva dos profissionais em relação a essas práticas é relevante para o estímulo no uso das PIC pelos usuários $^{15}$.

Outro fato que chama a atenção está relacionado à expansão das PIC no SUS. Entretanto, esse aumento foi mais expressivo a partir da aplicação das práticas por profissionais não médicos ${ }^{45}, \mathrm{o}$ que exige de outros membros da equipe a ampliação do conhecimento sobre os tratamentos complementares e a conquista do espaço para a utilização de tais práticas ${ }^{8}$. Para isso, é importante e necessário apoio e incentivo de gestores na oferta desses recursos, de modo a resgatar a dimensão humanística do atendimento em saúde ${ }^{13}$.

Nesse contexto, a ampliação dos saberes sobre a política e as PIC, bem como o incentivo aos profissionais, por meio, por exemplo, de educação permanente, podem ser estratégias eficazes na concretização e ampliação da implantação da PNPIC e melhoria do acesso às práticas nos serviços de saúde no âmbito do SUS. 
Mesmo que ainda existam poucas pesquisas brasileiras sobre a educação permanente voltada para essas práticas, Santos e Tesser ${ }^{46}$ apresentam um método de implantação e promoção de acesso às $\mathrm{PIC}$ na $\mathrm{ABS}$, baseado em experiências prévias, constituído de quatro fases sequenciais. A Primeira fase estabelece as pessoas responsáveis, que irão conduzir esse processo (preferencialmente profissionais com experiência em PIC). Na Segunda fase será realizada uma análise situacional, em que esses profissionais, atuantes ou não, serão mapeados e recrutados para que, por meio de discussões de implantação e acesso, realizem um levantamento sobre questões que dificultam o acesso às PIC, sobre as estratégias de organização das ações, sobre o fluxo de atendimento dos serviços e sobre a formalização das ações desenvolvidas, fazendo uma análise situacional local das PIC.

Durante a Terceira fase ocorrerá a regulamentação, estabelecendo-se as normas e adequações para o desenvolvimento das PIC em consonâncias com a política vigente (PNPIC) e, por fim, durante a Quarta fase, acontece a implantação, realizada de modo cíclico e contínuo, que será influenciada pela capacidade produtiva do pessoal responsável ${ }^{46}$.

Esse modelo pode auxiliar gestores e profissionais de maneira estratégica na ampliação de serviços já existentes ou implementação de novos serviços que facilitarão e/ou permitirão o acesso da população em geral às PIC. Com isso, é provável que sejam necessários investimentos iniciais e capacitação contínua dos profissionais da rede, com o objetivo de atender a demanda de modo qualificado e resolutivo. Contudo, com o passar do tempo, pode haver redução dos valores e maior qualidade de atendimento nos serviços, visto que a maioria das práticas necessita de baixo custo operacional e apresenta resultados rápidos e satisfatórios.

\section{Principais avanços no uso das PIC e desafios futuros}

Apesar do aumento no uso das PIC nos últimos anos, o seu potencial terapêutico e suas contribuições para a saúde ainda são pouco explorados no SUS ${ }^{8,9}$. Mesmo que o MS tenha avaliado de modo positivo esse aumento ${ }^{47}$, existem lacunas, como a avaliações das PIC nos serviços e melhor acompanhamento do impacto causado pela política.

Além disso, a preeminência do modelo biomédico atual somado à tendência mercadológica na área da saúde, que transforma os saberes e práticas em mercadorias, pode ser uma importante limitação nos avanços esperados para essas práti$\mathrm{Ca}^{17}$. Desse modo, existe o desafio de aprofundar o cuidado em um modelo integral de assistência, superando a supremacia da lógica de serviços baseados na biomedicina ${ }^{18}$.

Outros desafios importantes estão relacionados à capacitação e ao incentivo dos membros da equipe de saúde, ao apoio aos profissionais não médicos, assim como a percepção e a compreensão das perspectivas das PIC. Estes fatores são essenciais para o sucesso da inserção das PIC no $\mathrm{SUS}^{20}$ e para que os princípios da PNPIC sejam assegurados, contribuindo para a promoção da saúde em toda rede de atenção ${ }^{22}$.

\section{Limitações do estudo e sugestões para estudos futuros}

A utilização de apenas dois descritores controlados ("Sistema Único de Saúde" e "Terapias complementares") pode ter reduzido o número de artigos avaliados quanto aos critérios de elegibilidade do estudo. Nesse sentido, para estudos futuros, sugere-se a inclusão de outros descritores mais específicos, como, por exemplo, "Níveis de atenção à saúde" ou "Atenção Primária à Saúde", além de especificar as PIC, especialmente as mais prevalentes, nos campos de busca (como a fitoterapia, a homeopatia, a acupuntura, as práticas corporais, entre outras), a fim de ampliar a gama de resultados obtidos.

\section{Considerações finais}

Considera-se, após uma década de implantação da política, que as PIC são oferecidas de forma incipiente no SUS e a escassez de dados sobre determinadas práticas mostram-se como uma limitação sobre o atual cenário dessa abordagem. Entretanto, é possível observar reflexos positivos para os usuários e para os serviços que aderiram à sua utilização, mesmo que ainda existam desafios em sua implementação, no seu acesso, no seu uso e na formação de profissionais capacitados.

Assim, são necessários novos estudos com abordagem histórica das práticas complementares após a criação da PNPIC e quais os impactos foram provocados na saúde pública brasileira, bem como o incentivo no aperfeiçoamento profissional, principalmente para os trabalhadores da atenção básica, como uma ferramenta fundamental para o sucesso da implantação, do acesso e do uso das PIC no SUS. 


\section{Colaboradores}

LO Ruela: projeto, concepção, análise e interpretação dos dados, redação do artigo e revisão crítica relevante do conteúdo intelectual, aprovação final da versão a ser publicada. CC Moura: análise e interpretação dos dados, redação do artigo e revisão crítica relevante do conteúdo intelectual, aprovação final da versão a ser publicada. CVC Gradim, J Stefanello, DH Iunes: análise e interpretação dos dados, redação do artigo e revisão crítica relevante do conteúdo intelectual, aprovação final da versão a ser publicada. RR Prado: análise e interpretação dos dados, revisão crítica relevante do conteúdo intelectual, aprovação final da versão a ser publicada.

\section{Referências}

1. Frass M, Strassl RP, Friehs H, Müllner M, Kundi M, Kaye AD. Use and acceptance of Complementary and Alternative Medicine among the general population and medical personnel: A Systematic Review. Ochsner J 2012; 12(1):45-56.

2. World Health Organization (WHO). WHO Traditional Medicine Strategy 2002 - 2005. Geneva: WHO; 2002.

3. Brasil. Ministério da Saúde (MS). Política Nacional de Práticas Integrativas e Complementares no SUS. Brasília: MS; 2006.

4. Brasil. Ministério da Saúde (MS). Revista Brasileira Saúde da Família. Práticas Integrativas e Complementares em Saúde: uma realidade no SUS. Brasília: MS 2008.

5. World Health Organization (WHO). WHO traditional medicine strategy: 2014-2023. Geneva: WHO; 2013.

6. Whittemore R, Knafl K. The integrative review: updated methodology. J Adv Nurs 2005; 52(5):546-553.

7. Ursi ES. Prevenção de lesões de pele no perioperatório: revisão integrativa da literatura [dissertação]. Ribeirão Preto: Universidade de São Paulo; 2005.

8. Ceolin T, Heck RM, Pereira DB, Martins AR, Coimbra VCC, Silveira DSS. A inserção das terapias complementares no sistema único de saúde visando o cuidado integral na assistência. Enferm. glob. 2009; 16:1-10.

9. Santos RL, Guimaraes GP, Nobre MSC, Portela AS Análise sobre a fitoterapia como prática integrativa no Sistema Único de Saúde. Rev. Bras. Pl. Med. 2011; 13(4):486-491.

10. Oliveira SGD, Moura FRR, Demarco FF, Nascente PS, Del Pino FAB, Lund RG. An ethnomedicinal survey on phytotherapy with professionals and patients from Basic Care Units in the Brazilian Unified Health System. J Ethnopharmacol 2012; 140(2):428-437.

11. Fontenele RP, Sousa DMP, Carvalho ALM, Oliveira FA. Fitoterapia na Atenção Básica: olhares dos gestores e profissionais da Estratégia Saúde da Família de Teresina (PI), Brasil. Cien Saude Colet 2013; 18(8):2385-2394.
12. Monteiro DA, Iriart JAB. Homeopatia no Sistema Único de Saúde: representações dos usuários sobre o tratamento homeopático. Cad Saude Publica 2007 ago; 23(8):1903-1912.

13. Salles SAC, Schraiber LB. Gestores do SUS: apoio e resistências à Homeopatia. Cad Saude Publica 2009; 25(1):195-202.

14. Galhardi WMP, Barros NF, Leite-Mor ACMB. O conhecimento de gestores municipais de saúde sobre a Política Nacional de Prática Integrativa e Complementar e sua influência para a oferta de homeopatia no Sistema Único de Saúde local. Cien Saude Colet 2013; 18(1):213-220.

15. Silva EDC, Tesser CD. Experiência de pacientes com acupuntura no Sistema Único de Saúde em diferentes ambientes de cuidado e (des)medicalização social. Cad Saude Publica 2013; 29(11):2186-2196.

16. Fontanella F, Speck FP, Piovezan AP, Kulkamp IC. Conhecimento, acesso e aceitação das práticas integrativas e complementares em saúde por uma comunidade usuária do Sistema Único de Saúde na cidade de Tubarão/SC. ACM 2007; 36(2):69-74.

17. Tesser CD, Barros NF. Medicalização social e medicina alternativa e complementar: pluralização terapêutica do Sistema Único de Saúde. Rev Saude Publica 2008; 42(5):914-920.

18. Andrade JT, Costa LFA. Medicina Complementar no SUS: práticas integrativas sob a luz da Antropologia médica. Saude Soc 2010; 19(3):497-508.

19. Marques LAM, Vale FVVR, Nogueira VAS, Mialhe FL, Silva LC. Atenção farmacêutica e práticas integrativas e complementares no SUS: conhecimento e aceitação por parte da população sãojoanense. Physis. 2011; 21(2):663-674.

20. Nagai SC, Queiroz MS. Medicina complementar e alternativa na rede básica de serviços de saúde: uma aproximação qualitativa. Cien Saude Colet 2011; 16(3):1793-1800.

21. Thiago SCS, Tesser CD. Percepção de médicos e enfermeiros da Estratégia de Saúde da Família sobre terapias complementares. Rev Saude Publica 2011; 45(2):249-257. 
22. Ischkanian PC, Pelicioni MCF. Desafios das práticas integrativas e complementares no SUS visando a promoção da saúde. Rev Brasileira de Crescimento Desenvolvimento Humano. 2012; 22(2):233-238.

23. Lima KMSV, Silva KL, Tesser CD. Práticas integrativas e complementares e relação com promoção da saúde: experiência de um serviço municipal de saúde. Interface (Botucatu) 2014; 18(49):261-272.

24. Sousa IMC, Tesser CD. Medicina Tradicional e Complementar no Brasil: inserção no Sistema Único de Saúde e integração com a atenção primária. Cad Saude Publica 2017; 33(1):e00150215.

25. Brasil. Ministério da Saúde (MS). Secretaria de Atenção à Saúde. Portaria no 145 , de 11 de janeiro de 2017. Altera procedimentos na Tabela de Procedimentos, Medicamentos, Órteses, Próteses e Materiais Especiais do SUS para atendimento na Atenção Básica. Diário Oficial da União 2017; 13 jan.

26. Brasil. Ministério da Saúde (MS). Secretaria de Atenção à Saúde. Portaria no 849 , de 27 de março de 2017. Inclui a Arteterapia, Ayurveda, Biodança, Dança Circular, Meditação, Musicoterapia, Neturopatia, Osteopatia, Quiropraxia, Reflexologia, Reiki, Shantala, Terapia Comunitária Integrativa e Yoga à Política Nacional de Práticas Integrativas e Complementares. Diário Oficial da União 2017; 28 mar.

27. Brasil. Ministério da Saúde (MS). Secretaria de Atenção à Saúde. Portaria no 702, de 21 de março de 2018. Altera a Portaria de Consolidação no 2/GM/MS, de 28 de setembro de 2017, para incluir novas práticas na Política Nacional de Práticas Integrativas e Complementares - PNPIC. Diário Oficial da União 2018; 22 mar.

28. Brasil. Ministério da Saúde (MS). Práticas Integrativas e Complementares no SUS: ampliação do acesso. Brasília: MS; 2009. [Informes da Atenção Básica No 53].

29. Kawakita K, Okada K. Acupuncture therapy: mechanism of action, efficacy, and safety: a potential intervention for psychogenic disorders? Biopsychosoc Med 2014; 8(1):4.

30. Zhao ZQ. Neural mechanism underlying acupuncture analgesia. Prog Neurobiol 2008; 85(4):355-375.

31. Carpenter JS, Neal JG. Other complementary and alternative medicine modalities: acupuncture, magnets, reflexology, and homeopathy. Am J Med 2005; 118(12B):109S-117S.

32. Cucherat M, Haugh MC, Gooch M, Boissel JP. Evidence of clinical efcacy of homeopathy. A meta-analysis of clinical trials. Eur J Clin Pharmacol. 2000; 56(1):27-33.

33. Iunes DH, Chaves ECL, Moura CC, Côrrea B, Carvalho LC, Silva AM, Carvalho EC. Role of Auriculotherapy in the treatment of temporomandibular disorders with anxiety in university students. Evid Based Complement Alternat Med 2015; 2015:430143.

34. Kurebayashi LFS, Gnatta JR, Borges TP, Belisse G, Coca S, Minami A, Souza TM, Silva MJP. Aplicabilidade da auriculoterapia com agulhas ou sementes para diminuição de estresse em profissionais de enfermagem. Rev Esc Enferm USP 2012; 46(1):89-95.
35. Macías-Cortés EC, Llanes-González L, Aguilar-Faisal L, Asbun-Bojalil J. Individualized Homeopathic Treatment and Fluoxetine for Moderate to Severe Depression in Peri- and Postmenopausal Women (HOMDEP-MENOP Study): A Randomized, Double-Dummy, Double-Blind, Placebo-Controlled Trial. PLoS One 2015; 10(3):e0118440.

36. Toca-Villegas J, Esmer-Sánchez D, García-Narváez J, Sánchez-Aguilar M, Hernández-Sierra JF. Eficacia de la auriculoterapia modificada como tratamento para el control del dolor post operatorio em pacientes intervenidos mediante colecistectomía laparoscópica. Cirurgia y Cirujanos (English Edition) 2017; 85(3):220-224.

37. Rossignol M, Bégaud B, Avouac B, Lert F, Rouillon F, Bénichou J, Massol J, Duru G, Magnier AM, Guillemot D, Grimaldi-Bensouda L, Abenhaim L. Who seeks primary care for musculoskeletal disorders (MSDs) with physicians prescribing homeopathic and other complementary medicine? Results from the EPI3-LASER survey in France. BMC Musculoskelet Disord 2011; 12:21.

38. Astin JA. Why patients use alternative medicine: results of a national study. JAMA 1998; 279(19):15481553.

39. Tesser CD. Práticas complementares, racionalidades médicas e promoção da saúde: contribuições poucos exploradas. Cad Saude Publica 2009; 25(8)1732-1742.

40. Brasil. Ministério da Saúde (MS). Politica Nacional de Atenção Básica. Brasília: MS; 2012.

41. Brasil. Ministério da Saúde (MS). Política Nacional de Práticas Integrativas e Complementares. Informe - maio de 2017 [Internet]. 2017 Maio [acessado 2018 Mar 06]. Disponível em: http://189.28.128.100/dab/ docs/portaldab/documentos/Informe_Maio_2017. pdf

42. Faqueti A, Tesser CD. Utilização de Medicinas Alternativas e Complementares na atenção primária à saúde de Florianópolis/SC: percepção de usuários. Cien Saude Colet 2016; 23(8):2621-2630.

43. Brasil. Ministério da Saúde (MS). Departamento de Atenção Básica. Portal da Saúde SUS. Portaria amplia oferta de PICS [Internet]. 2017 Jan [acessado 2017 Jul 09]. Disponível em: http://dab.saude.gov.br/portal$\mathrm{dab} /$ noticias.php? conteudo=_\& $\operatorname{cod}=2297$

44. Spadacio C, Castellanos MEP, Barros NF, Alegre SM, Tovey P, Broom A. Medicinas Alternativas e Complementares: uma metassíntese. Cad Saude Publica 2010; 26(1):7-13.

45. Sousa IMC, Bodstein RCA, Tesser CD, Santos FAZ, Hortale VA. Práticas integrativas e complementares: oferta produção de atendimentos no SUS e em municípios selecionados. Cad Saude Publica 2012; 28(11):2143-2154.

46. Santos MC, Tesser CD. Um método para a implantação e promoção de acesso às Práticas Integrativas e Complementares na Atenção Primária à Saúde. Cien Saude Colet 2012; 17(11):3011-3024.

47. Brasil. Ministério da Saúde (MS). Relatório de Gestão 2006/2010 - Práticas Integrativas e Complementares no SUS. Brasília: MS; 2011.

Artigo apresentado em 19/07/2017

Aprovado em 20/04/2018

Versão final apresentada em 22/04/2018 